\title{
Predicting Excavation-Induced Tunnel Response by Process-Based Modelling
}

\author{
Linlong Mu $\mathbb{D}^{1,2}$ Jianhong Lin, ${ }^{1,2}$ Zhenhao Shi $\mathbb{D}^{1,2}$ and Xingyu Kang ${ }^{1,2}$ \\ ${ }^{1}$ Department of Geotechnical Engineering, Tongii University, Shanghai 200092, China \\ ${ }^{2}$ Key Laboratory of Geotechnical and Underground Engineering of Ministry of Education, Tongji University, \\ Shanghai 200092, China \\ Correspondence should be addressed to Zhenhao Shi; 1018tjzhenhao@tongji.edu.cn
}

Received 17 December 2019; Revised 19 March 2020; Accepted 3 April 2020; Published 28 April 2020

Guest Editor: Tomas Veloz

Copyright $\odot 2020$ Linlong Mu et al. This is an open access article distributed under the Creative Commons Attribution License, which permits unrestricted use, distribution, and reproduction in any medium, provided the original work is properly cited.

Potential damages to existing tunnels represent a major concern for constructing deep excavations in urban areas. The uncertainty of subsurface conditions and the nonlinear interactions between multiple agents (e.g., soils, excavation support structures, and tunnel structures) make the prediction of the response of tunnel induced by adjacent excavations a rather difficult and complex task. This paper proposes an initiative to solve this problem by using process-based modelling, where information generated from the interaction processes between soils, structures, and excavation activities is utilized to gradually reduce uncertainty related to soil properties and to learn the interaction patterns through machine learning techniques. To illustrate such a concept, this paper presents a simple process-based model consisting of artificial neural network (ANN) module, inverse modelling module, and mechanistic module. The ANN module is trained to learn and recognize the patterns of the complex interactions between excavation deformations, its geometries and support structures, and soil properties. The inverse modelling module enables a gradual reduction of uncertainty associated with soil characterizations by accumulating field observations during the construction processes. Based on the inputs provided by the former two modules, the mechanistic module computes the response of tunnel. The effectiveness of the proposed process-based model is evaluated against high-fidelity numerical simulations and field measurements. These evaluations suggest that the strategy of combining artificial intelligence techniques with information generated during interaction processes can represent a promising approach to solve complex engineering problems in conventional industries.

\section{Introduction}

With the rapid development of cities, it becomes frequent that deep excavations constructed for the foundation of high-rise buildings and/or underground space are next to existing subway or sewage tunnels [1-4]. As illustrated in Figure 1, the stress releases caused by excavations inevitably result in ground movements that can subsequently cause the deformations of tunnels and structural damages such as lining cracks and joint dislocations [5]. These problems can impair the operation of subways/sewage systems and even cause civilian casualties. Therefore, it is critical for engineers to accurately predict the response of tunnels subjected to adjacent deep excavations.
Uncertainty is the distinct feature of geotechnical engineering in that soils and rocks created by natural processes are variable in their composition, behaviour, and distributions. Such variability of the subsurface conditions, combined with the nonlinear interactions between multiple agents (e.g., soils, excavation support structures, construction activities, and tunnel structures), make the prediction of excavation-induced tunnel response a rather complex and difficult task. The latter is currently solved normally by involving numerical modelling techniques associated with advanced soil constitutive models $[1,6,7]$. Nevertheless, constructing a numerical model that can generate reasonable outcomes requires considerable expertise in numerical modelling and a systematic material characterization 


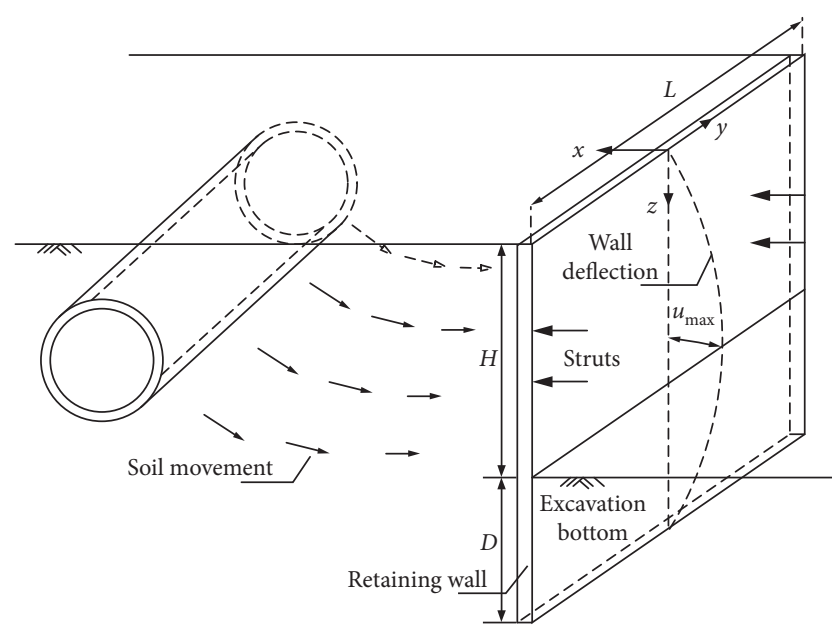

FIGURE 1: Schematics showing the influence of deep excavations on adjacent tunnels.

program to calibrate soil models. These prerequisites, quite possibly, are unrealistic for many civil engineers. There is another reason why an alternative strategy is needed to analyse the problem. In the last decade, building information modelling (BIM) has shown its potential in transforming the construction industry by facilitating the digitalization and automation of the full-lifecycle operations (i.e., design, construction, and maintenance) of buildings and infrastructures $[8,9]$. Accordingly, a large amount of data pertaining to different stages of structures is collected by BIM platforms. To exploit such big data and enable dynamic and automated managements of excavation construction, analysis models that are capable of self-learning and computationally efficient are necessary.

This work proposes an initiative to devise an adaptive and efficient solution to the engineering problem discussed above, by harnessing the power generated by process-based modelling, where information generated during construction processes is used to reduce the uncertainty associated with subsurface conditions and recognize the interaction patterns between soils, structures, and excavation activities, so that the response of adjacent tunnels can be accurately predicted. To proof this concept, here we build a simple process-based model consisting artificial neural network (ANN) module, inverse modelling module, and mechanistic module. As will be described in the following, the mechanistic component is responsible for computing the response of tunnels under given deformations of excavation support structures. The latter cannot be easily obtained from conventional mechanical models, as they are influenced by a large number of variables, including excavation geometries, excavation support structures, and soil mechanical properties. Here, we exploit ANN's advantages in recognizing patterns of complex interactions involving multiple factors $[10,11]$ and use it to predict the deformations of excavation support structures. To further reduce the uncertainty associated with the soil properties fed into the ANN model, the module of inverse modelling [12-14] is utilized to search for an optimal set of soil parameters that can minimize the difference between measured deformations of excavation support structures and those predicted by ANN. The advantage of collaborating the two machine learning techniques for engineering practice is reflected in that the inverse modelling will use field measurements at the early stages of construction processes to optimize soil parameters, thus consequently improving the predictions of the ANN model for later and more critical stages of the same project. The development of ANN models in the field of civil engineering normally faces the obstacle of lacking field data for model training. Aimed at constructing a platform to explore the potential of ANN models in civil engineering practices, here we train the ANN model by synthetic cases generated by numerical simulations. As will be shown in the following, such ANN model can provide useful information for realworld applications.

The rest of the paper is organized as follows. We will first present the mechanistic model used to compute the response of tunnels, followed by a description of the construction, training, and validation of the ANN model, as well as the inverse modelling module. Finally, the performance of the proposed hybrid model is evaluated against field measurements obtained from an excavation project next to the tunnels of a subway in China.

\section{Mechanistic Model for Computing Response of Tunnel due to Adjacent Excavations}

We formulate the mechanistic model based on the strategy of two-stage analysis [4, 15-17], where free-field soil movements or stress changes caused by excavation (i.e., the soil displacements and stress releases that would occur without the presence of tunnels) are first estimated, and the response of tunnel is subsequently evaluated by idealizing the tunnel structure as an elastic beam sitting either on nonlinear spring (i.e., the so-called Winkler subgrade reaction hypothesis) or elastic continuum [17, 18] and by imposing the displacements or stresses fields obtained from the first stage. Here, we build a displacement-controlled twostage model (see Figure 1), as it can deliver more accurate predictions in particular for shallowly embedded tunnels where the presence of tunnels can significantly alter ground stress fields but soil displacements to a limited extent.

2.1. Free-Field Soil Movements Induced by Excavation. A crucial element in the two-stage model is to estimate the free-field soil movements caused by excavations. Numerous studies have indicated that appropriate consideration of small-strain behaviour of soils (i.e., considerably high stiffness at very small-strain levels and a nonlinear stiffness degradation with strain magnitudes) is essential for correctly predicting the soil movements associated with the construction activities of braced excavations [19-21]. Accordingly, we adopt the following closed-form expressions for the free-field soil movements proposed by $\mathrm{Mu}$ and Huang [22], which are obtained by fitting finite element (FE) simulations with a soil constitutive model that considers small-strain behaviour: 


$$
\begin{aligned}
& u_{s}(x, y, z)= u_{\max } \cdot a_{x} \cdot e^{-9((z-H) /(H+D))^{2}-\pi(y / R)^{2}} \cdot e^{-\left(\left((x / z)-b_{x}\right) / c_{x}\right)^{2},} \\
& w_{s}(x, y, z)= \begin{cases}0.8 u_{\max } \cdot a_{z} \cdot\left(\frac{x}{H}+0.5\right) \cdot e^{-\pi(y / R)^{2}-\left(\left((z / x)-b_{x}\right) / c_{x}\right)^{2},} & 0 \leq x \leq 0.5 H, \\
0.8 u_{\max } \cdot a_{z} \cdot\left(-0.6 \frac{x}{H}+1.5\right) \cdot e^{-\pi(y / R)^{2}-\left(\left((z / x)-b_{x}\right) / c_{x}\right)^{2}}, & 0.5 H \leq x \leq 2 H, \\
0.8 u_{\max } \cdot a_{z} \cdot\left(-0.05 \frac{x}{H}+0.2\right) \cdot e^{-\pi(y / R)^{2}-\left(\left((z / x)-b_{x}\right) / c_{x}\right)^{2},} & 2 H \leq x \leq 4 H,\end{cases}
\end{aligned}
$$

where $u_{s}$ and $w_{s}$ denote the soil movements along the vertical direction and horizontal direction perpendicular to the retaining wall (the horizontal displacements parallel to the wall are often negligible); $x, y$, and $z$ are spatial coordinates of soils (see Figure 1); and the variables $L$ and $H$ denote the length and depth of the excavation, respectively, $D$ is the embeded depth of the retaining wall, while $R=L\left[\begin{array}{ll}0.069 & \ln (H / L)+1.03\end{array}\right] / 2$ is a composite geometry factor. The coefficient $a_{x}, b_{x}, c_{x}, a_{z}, b_{z}$, and $c_{z}$ are functions of soil spatial coordinates and excavation geometries, and their specific expressions can be found in $\mathrm{Mu}$ and Huang [22]. It should be emphasized that the soil displacement field of equation (1) is a function of the maximum deflection of earth retaining wall $u_{\max }$ defined in Figure 1 . The latter quantity will be predicted by the ANN model described later.

\subsection{Tunnel Response Subjected to Prescribe Soil Displacements.} With the soil displacement fields described above, we further evaluate the tunnel response by idealizing it as an elastic beam that complies with Euler-Bernoulli beam theory:

$$
\begin{aligned}
\frac{\mathrm{d}^{4} u}{\mathrm{~d} y^{4}}+\frac{k}{E I}\left(u-u_{s}\right) & =0, \\
\frac{\mathrm{d}^{4} w}{\mathrm{~d} y^{4}}+\frac{k}{E I}\left(w-w_{s}\right) & =0,
\end{aligned}
$$

where $u$ and $w$ are the vertical and horizontal displacements of the tunnel, $u_{s}$ and $w_{s}$ are the soil displacements prescribed by equation (1), EI is the bending stiffness of tunnel, and $y$ is the axial direction of the tunnel. The variable $k$ in equation (2) denotes modulus of subgrade reaction (i.e., the stiffness of the springs connected to the beam, see Figure 2). To consider the influences of the burial depth and size of tunnel on soil reactions, the expression proposed by $\mathrm{Yu}$ et al. [23] is adopted here:

$$
k=\frac{3.08}{\eta} \frac{E_{s}}{1-v_{s}^{2}} \sqrt[8]{\frac{E_{s} B}{E I}} .
$$

In equation (3), $B$ is the tunnel diameter, $E_{s}$ and $\nu_{s}$ are Young's modulus and Poisson's ratio of soils, and $\eta$ is a correction coefficient that depends on the burial depth of tunnel $z$ :

$$
\eta= \begin{cases}2.18, & z / B \leq 0.5, \\ 1+\frac{1}{1.7(z / B)}, & z / B>0.5 .\end{cases}
$$

We use the finite difference method to solve the governing equation of equations (2) and (3). As depicted in Figure 2, the tunnel along its longitudinal axis is equally divided into small beam elements with their lengths given by $h=S / n$, where $n$ is the number of elements and $S$ is the length of the tunnel. As equation (2) involves fourth-order derivatives, two virtual joints are added to both ends of the tunnel so that equation (2) can be approximated by the central difference scheme:

$$
w_{i+2}-4 w_{i+1}+6 w_{i}-4 w_{i-1}+w_{i-2}=4 \lambda^{4} h^{4}\left(w_{s i}-w_{i}\right)
$$

where $\lambda=\sqrt[4]{k / 4 E I}$ is the flexibility eigenvalue of the beam equation and the assembly of the governing equation for each beam elements leads to the following discretized form of the equation of motions for the tunnel:

$$
[K]\{w\}=\{F\},
$$

where the vector $\{w\}=\left[w_{0}, w_{1}, \ldots, w_{i}, \ldots, w_{n-1}, w_{n}\right]$ contains the nodal displacements of the tunnel, while $\{F\}=$ $\left[k w_{s(0)}, k w_{s(1)}, \ldots, k w_{s(i)}, \ldots, k w_{s(n-1)}, k w_{s(n)}\right]$ contains the corresponding nodal force determined under the assumption that shear forces and moments acting on both ends of the tunnel are negligible (i.e., the axial dimension of the tunnel is much greater than the size of the excavation). The matrix $[K]$ in equation (6) is the generalized system stiffness expressed as 


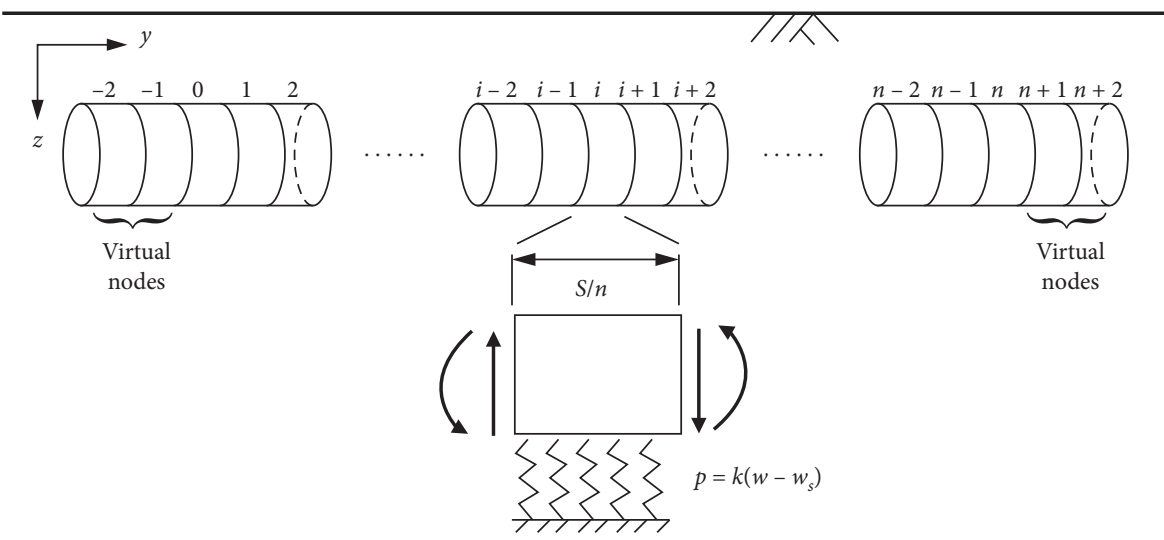

Figure 2: Schematics showing the idealization of tunnel structure as an elastic beam and the simplification of tunnel-soil interaction as beam sitting on elastic springs.

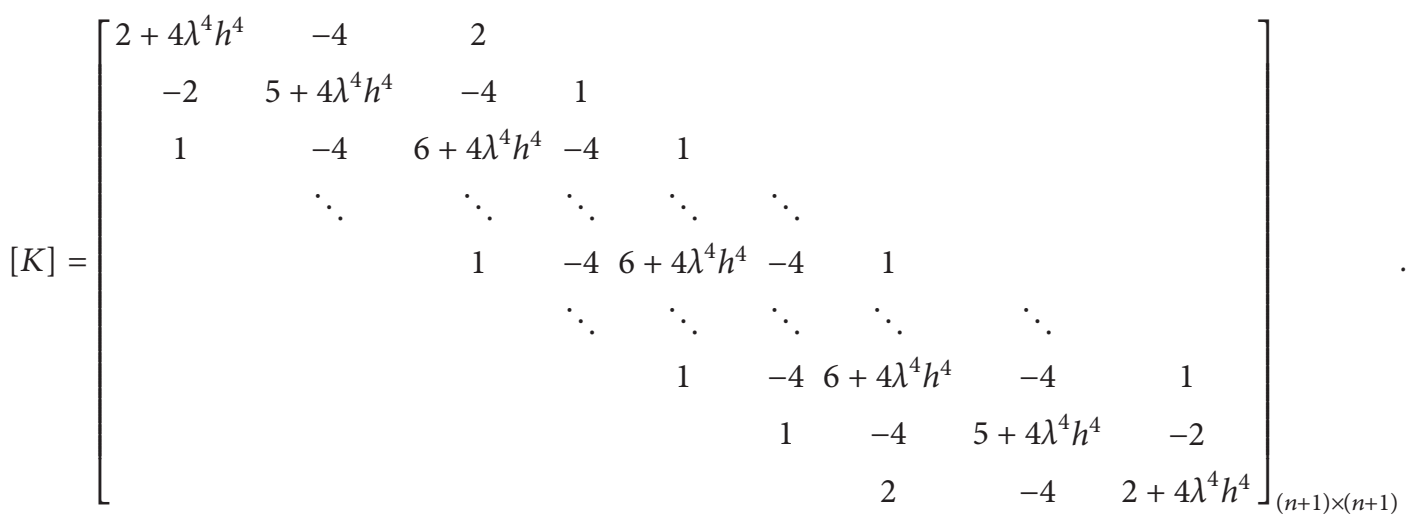

\subsection{ANN Model for Predicting Retaining Wall Deflection.} The key input of the mechanistic model described in the previous section is the maximum deflection of earth retaining wall $u_{\max }$ (i.e., see equation (1)). The ANN model aimed at predicting this quantity, including the structure of the neural network, its training, and validation, is discussed as follows.

2.3.1. Neural Network Structure. An ANN model generally has a layered structure consisting of input layer, hidden layers, and output layer [10]. The input layer contains parameters that are relevant to the problem at hand and can be determined relatively reliably, while the output layer contains information that is normally unknown and to be predicted by the ANN model. The input and output layers are connected by intermediate hidden layers made up of nodes interconnected to each other. Differential weights (i.e., strength) can be assigned to the connections between nodes to reflect different patterns of the correspondence between input parameters and output variables.

The input layer of our ANN model is presented in Figure 3 and Table 1. These parameters are selected based on their influences on the deflections of earth retaining wall and whether they can be altered during the construction (i.e., to dynamically optimize the original design). These attributes can be categorized into three groups (see), including excavation geometries, mechanical properties of excavation support structures, and soil properties. To digitalize soil properties into forms suitable for the ANN model, we adopt the hardening soil small (HSS) model [24] as a theoretical template to describe the mechanical characters of soils, as such constitutive model explicitly incorporates small-strain behaviour and is extensively validated by engineering practice $[25,26]$. The HSS model has 8 mechanical parameters, which add the unit weight of soil and soil layer thickness up to 10 parameters for each soil stratum. The number of inputs for an ANN model is normally fixed, which poses difficulty for applying the same ANN model for projects with different numbers of soil layers. To address this issue, we divide soils within the range of retaining wall (i.e., the portion of soils that can significantly impact the retaining wall deflections) into a given number of artificial soil layers (currently 10 layers are employed) in that an actual soil stratum is represented by multiple sublayers with the same soil properties.

The finally constructed neural network has an input layer of 111 parameters that are connected to a single output of retaining wall deflection by two hidden layers. Each of these hidden layers has 30 nodes to ensure the generalizability of the model.

2.3.2. Neural Network Training. To help the ANN model correctly recognize the patterns of the interactions between excavation support structures and surrounding soils, a large 


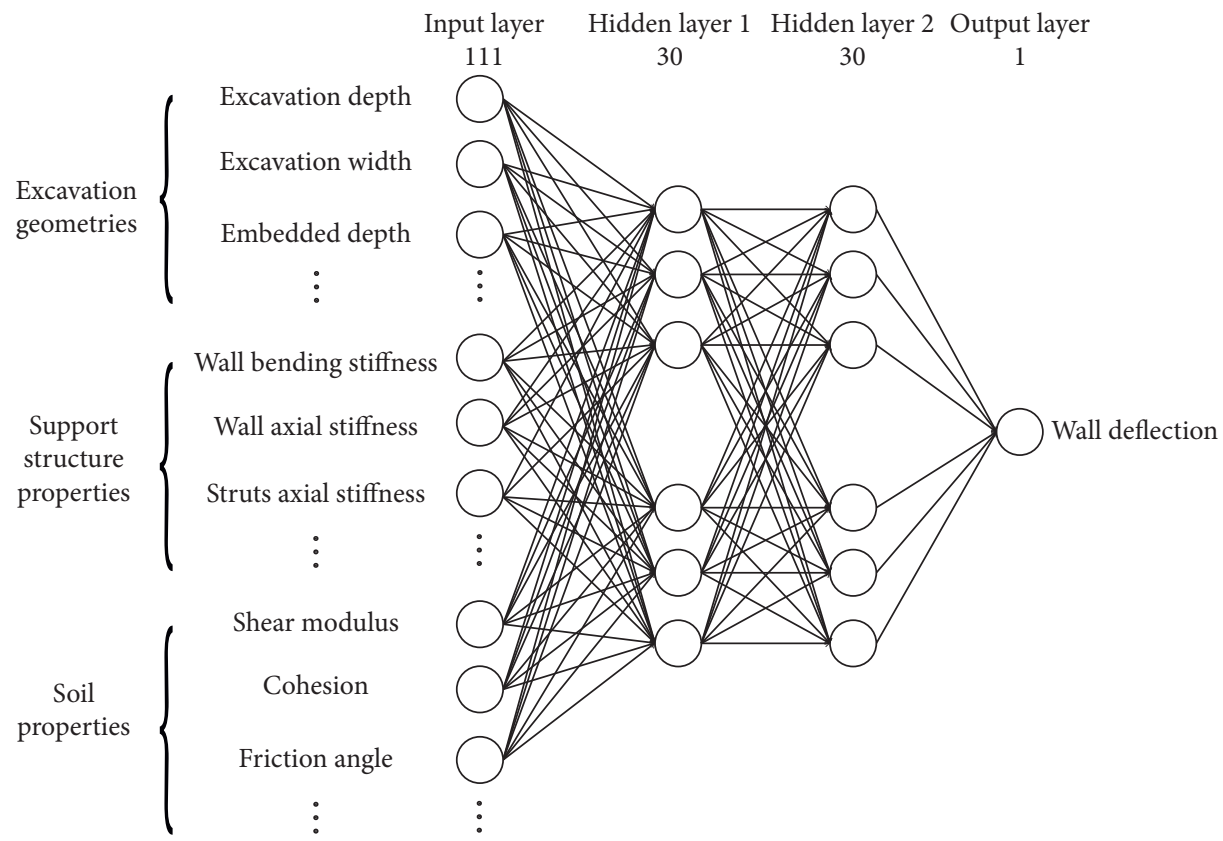

FIGURE 3: Layered structure of the proposed neural network.

TABLE 1: ANN input layer.

\begin{tabular}{lcc}
\hline Excavation geometries & $\begin{array}{c}\text { Mechanical properties of support } \\
\text { structures }\end{array}$ & $\begin{array}{c}\text { Soil properties (parameterized through } \\
\text { HSS model) }\end{array}$ \\
\hline $\begin{array}{l}\text { Excavation depth } \\
\text { Excavation width }\end{array}$ & $\begin{array}{c}\text { Bending stiffness of retaining wall, } \\
\text { Axial stiffness of retaining wall, EA } \\
\text { Embedded depth of retaining wall }\end{array}$ & $\begin{array}{c}\text { Shear modulus at very small strains } \\
\text { Poisson's ratio of retaining wall } \\
\text { material }\end{array}$ \\
$\begin{array}{l}\text { Excavation depth divided by the layers of struts } \\
\text { Vertical distance between excavation bottom and the } \\
\text { nearest lateral support } \\
\text { Averaged vertical and lateral space between struts }\end{array}$ & Axial stiffness of struts, EA & Angle of internal friction \\
\hline
\end{tabular}

number of reliable samples are required to train the model. Ideally, the types of data used to train the ANN model shall be the same as those involved while utilizing ANN to actually predict. Accordingly, the best training information comes from actual engineering projects where the ANN model is intended to apply, including accurate descriptions of excavation geometries, the sizes and materials of support structures, the HSS model parameters for each of soils layers encountered, and finally a reliable measurement of retaining wall deflections. Unfortunately, reported case studies that contain both information pertaining to the inputs and outputs of the ANN model is currently not enough for training the model. As a workaround, we train the ANN model through synthetic samples generated by two-dimensional finite element (FE) simulations. Training our ANN model by synthetic examples leads to two additional advantages. First, the generated datasets can cover the wide ranges of each input attribute (e.g., the ranges of excavation depth, soil strength, and structure stiffness) possibly encountered in engineering practice. Second, compared with actual field data, the datasets generated by numerical simulations tend to be free of noises associated with factors excluded by the ANN model (like man power and the management levels), thus helping the ANN concentrate on the correlation between its inputs and outputs.

Totally, 1943 synthetic samples are generated by varying ANN input parameters randomly but within typical ranges encountered in engineering practice (e.g., the space between lateral braces varies from $3 \mathrm{~m}$ to $12 \mathrm{~m}$ and excavation length varies from $20 \mathrm{~m}$ to $80 \mathrm{~m}$ ). Examples of employed training samples (note that soil parameters are excluded due to space limitations) are shown in Table 2.

We use the backpropagation algorithm coded in PyTorch module to train our ANN model, and this supervised learning process involves five epochs of forward activation towards outputs and backward errors propagation of adjusting connection weights. The activation function utilized for ANN nodes is rectified linear unit ( $\mathrm{ReLu}$ ), the optimization method is Adam, and the cost function is average sum-of-squares error (MSE). Among all synthetic samples, $70 \%$ is used to train the ANN model, while the rest is reserved as the validation set used by the learning algorithm. 
TABLE 2: Examples of synthetic samples employed to train the ANN model.

\begin{tabular}{lcccccccc}
\hline \multicolumn{2}{c}{$\begin{array}{l}\text { Excavation } \\
\text { geometries }\end{array}$} & \multicolumn{3}{c}{ Retaining wall properties } \\
$\begin{array}{l}\text { Depth } \\
(\mathrm{m})\end{array}$ & $\begin{array}{c}\text { Width } \\
(\mathrm{m})\end{array}$ & $\begin{array}{c}E I \\
\left(10^{6} \mathrm{kN} \times \mathrm{m}^{2} / \mathrm{m}\right)\end{array}$ & $\begin{array}{c}E A \\
\left(10^{6} \mathrm{kN} / \mathrm{m}\right)\end{array}$ & $\begin{array}{c}\text { Embedded depth } \\
(\mathrm{m})\end{array}$ & $\begin{array}{c}\text { Vertical space } \\
(\mathrm{m})\end{array}$ & $\begin{array}{c}\text { Lateral space } \\
(\mathrm{m})\end{array}$ & $\begin{array}{c}E A \\
\left(10^{6} \mathrm{kN}\right)\end{array}$ & $\begin{array}{c}\text { Wall deflection } \\
(\mathrm{mm})\end{array}$ \\
\hline 4.9 & 40 & 1.507 & 18.1 & 30.1 & 4.0 & 4 & 3 & 1.33 \\
20 & 40 & 1.507 & 18.1 & 15.0 & 2.9 & 3 & 3 & 3 \\
5 & 60 & 1.667 & 20.0 & 30.0 & 3.6 & 4 & 2 & 5.39 \\
21 & 80 & 1.667 & 20.0 & 14.0 & 4.23 & 9 & 16 & 46.20 \\
\hline
\end{tabular}

2.3.3. Neural Network Validation. To assess the performance of the trained model, we compare the retaining wall deflections predicted by ANN and those computed by FE simulations for 46 different combinations of variables other than those used for synthetic samples. Figure 4 shows that the maximum wall deflection predicted by the ANN model reasonably agrees with that calculated by the high-fidelity numerical simulations, with the mean relative error of $12.2 \%$. The ANN model is further examined against data from three actual excavation projects [27-29], where soil parameters and wall defection measurements are all reported. As shown in Table 3, the model predictions and field measurements match reasonably. The latter fact suggests that although the ANN model is trained by synthetic samples, it is capable of predicting the deformations of excavation support structures in actual engineering practice.

2.4. Inverse Model for Reducing Uncertainty with Soil Parameters. Among all input parameters of the constructed ANN model, those related to the mechanical properties of soils have the highest degree of uncertainty attributed to natural variations of soils and the disturbance effects associated with coring, transporting, and trimming soil specimens before they are tested in laboratories. On the contrary, while soils in the field preserve the highest integrity, currently there lacks theoretical frameworks capable of estimating soil constitutive parameters by directly interpreting in situ measurements. To more accurately determine soil parameters and predict retaining wall deflections, we combine the proposed ANN model with inverse modelling, which automatically searches for accurate descriptions of soil properties by minimizing the difference between measured retaining wall deflections and those computed by the ANN model. The most important advantage of collaborating the two machine learning techniques for engineering practice is that the inverse modelling can use field measurements at early stages of construction activities to optimize soil parameters, thus improving the predictions of the ANN model for later and more critical stages of the same project.

The inverse modelling is achieved here through a nonlinear optimization algorithm based on an objective function of the least-squares form:

$$
S=\sum_{i}^{m}\left(y_{i}-y_{i}^{\prime}(b)\right)^{2} \omega_{i},
$$

where $b$ contains the soil parameters to be determined; $y$ and $y^{\prime}$ denote the vectors of field observations and ANN

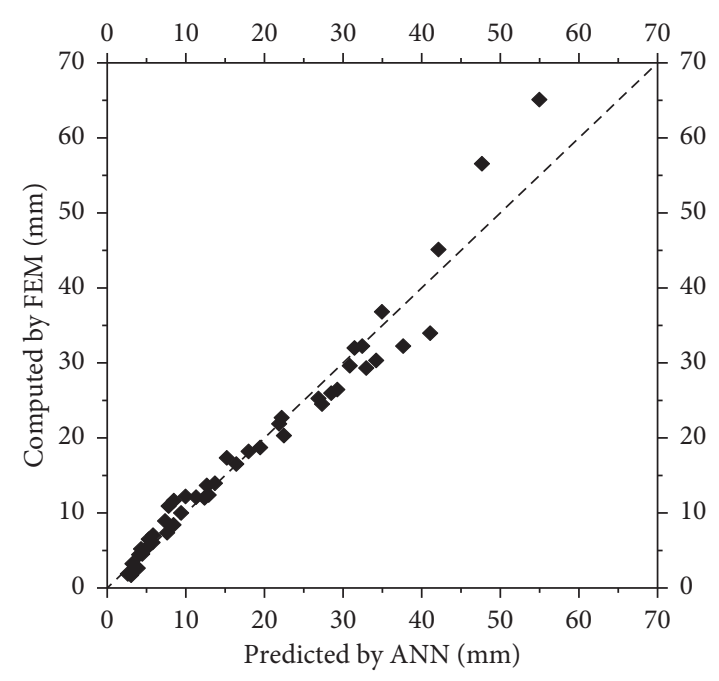

FIGURE 4: Comparison between maximum retaining wall deflections computed by finite element simulations and predicted by the ANN model.

predictions, respectively; the number of observation response is $m$; the symbol $\omega_{i}$ denotes weight assigned to different observations based on their reliability and accuracy and here given by the inverse of the error variance that corresponds to each observation, i.e., $\omega_{i}=1 / \sigma_{i}^{2}$ [13]. An optimal set of soil parameters that minimizes the objective function of equation (9) is automatically searched by a modified Gauss-Newton algorithm with additional damping and Marquardt parameters [30, 31].

We use two statistic indexes related to the optimization algorithm to determine whether certain soil parameters can be accurately and uniquely determined through the inverse modelling scheme described above:

$$
\mathrm{CSS}_{j}=\sqrt{\frac{1}{m} \sum_{i=1}^{m}\left(\frac{\partial y_{i}^{\prime}}{\partial b_{j}} b_{j} \sqrt{\omega_{i}}\right)^{2}}
$$

$\mathrm{CSS}_{j}$ is the so-called composite scaled sensitivity used to indicate the degree of the overall influences of the $j^{\text {th }}$ soil parameters on all observable responses $(i=1$ to $m)$ selected entering into the inverse model, such as retaining wall deflections. It should be emphasized that even for the same parameters, CSS can vary when different field observations are involved, and therefore, the value of CSS can effectively identify the possibility of reducing the uncertainty associated 
TABLE 3: Comparison between measured maximum deflections of retaining wall in case studies and ANN predictions.

\begin{tabular}{lccr}
\hline Case number & 1 & 2 & 3 \\
\hline Measurements (mm) & 48.30 & 20.40 & 23.52 \\
ANN predictions (mm) & 47.60 & 23.35 & 20.76 \\
Relative error (\%) & 1.45 & 14.45 & 11.72 \\
\hline
\end{tabular}

with particular parameters with respect to given types of field measurement. The second useful statistic index is the parameter correlation coefficient:

$$
\mathrm{PCC}_{i j}=\frac{V_{i j}}{\sqrt{V_{i i} V_{j j}}},
$$

which measures the degree of correlation between the $i^{\text {th }}$ and $j^{\text {th }}$ parameters. A pair of parameters that are highly correlated to each other, indicated by high PCC values, usually cannot be simultaneously determined through the inverse modelling scheme described above, as the uniqueness of the solution cannot be ensured. $V_{i j}$ in equation (10) denotes the component of the variance-covariance matrix:

$$
[V]=\frac{S}{m-n}\left(\left[\frac{\partial y^{\prime}}{\partial b}\right]^{T}[\omega]\left[\frac{\partial y^{\prime}}{\partial b}\right]\right)^{-1},
$$

where $n$ denotes the number of parameters to be determined; $S$ is the value of the objective function defined in equation (8); and $\left[\partial y^{\prime} / \partial b\right]$ is a matrix containing the gradient of the observation with respect to soil parameters, while $[\omega]$ is a diagonal matrix with its component given by $\omega_{i}$ defined above.

\section{Evaluation of Mechanistic-Machine Learning Hybrid Model}

3.1. Determining Input Parameters for ANN Model. The excavation project located in Hangzhou, China [32], provides an unique opportunity to assess the proposed model for analyzing the response of tunnel subjected to adjacent excavation. In this case study, the deep excavation extends to the depths of $15 \mathrm{~m}$ to $18.55 \mathrm{~m}$ below ground surface and is beside the tunnels of a subway's north and south lines. The smallest distance between the excavation and the tunnels is only $7 \mathrm{~m}$, while the averaged horizontal space is around $9 \mathrm{~m}$. The length of the excavation parallel to the tunnels is $245 \mathrm{~m}$. The depth of the tunnel crown varies from $9.3 \mathrm{~m}$ to $13.3 \mathrm{~m}$, and the diameter of the tunnel is $6.2 \mathrm{~m}$. To reduce the excavation-induced impacts on the tunnels, the part of the excavation next to the tunnels adopts a composite retaining wall of $1000 \mathrm{~mm}$ thick diaphragm wall and cast-in-place piles, and the embedded depth of the retaining wall is $6 \mathrm{~m}$. The internal supports consist of three layers of reinforced concrete struts. According to these in situ conditions, the geometries of the excavation that are fed into the ANN model are obtained (i.e., see those depicted in Figure 5, note that the width of the excavation is $70 \mathrm{~m}$ and the horizontal space between struts is $6 \mathrm{~m}$ ). The mechanical properties of excavation support structures used in the ANN model are summarized in Table 4, as reported in the case study. Here, we increase the thickness of the diaphragm wall to $1500 \mathrm{~mm}$ to consider the strengthening effects due to the additional cast-in-place piles.

The subsurface at the site is composed of three strata, among which the first layer is categorized as sandy silt (SM), while the second and third layers are clayey silt $(\mathrm{CM})$, as shown in Figure 5. Table 5 summaries the hardening soil small parameters for the first two layers (i.e., those within the range of the retaining wall) calibrated from laboratory measurement [32]. These soil properties are adopted in the ANN model.

3.2. Model Performance. Figure 6 compares the measured deflection profiles of retaining wall against the maximum deflections predicted by the ANN model that corresponds to two consecutive stages of the excavation (i.e., excavate to $13 \mathrm{~m}$ and $16 \mathrm{~m}$ depths). Because of the large number of variables involved in this problem, we consider the ANN prediction still acceptable, although it tends to overestimate the wall deformations. The maximum discrepancy is around $8 \mathrm{~mm}$ occurred for the excavation to the shallower depth, and the prediction is improved as the construction proceeds in that the difference between measured and predicted maximum wall deflection reduces to $5 \mathrm{~mm}$ as the excavation reaches $16 \mathrm{~m}$.

Among the resources that may result in the mismatches described above, the uncertainty embedded with the characterization of soil properties is a primary contributor. The fact that the deformations of the excavation support structures predicted by the ANN model are greater than those observed suggests the possibility that soil specimens have been disturbed and consequently weakened before they are tested. To minimize the influences of the uncertainty associated with the parameters for soils, we employ the inverse modelling described in the previous section. To constrain the number of parameters to be determined and consequently enhance inverse modelling performance, for each layer we only directly optimize two parameters, the reference stiffness modulus $E_{50}$ and the reference shear strain threshold $\gamma_{0.7}$, i.e., one controls the soil behaviour at large smalls and the other for small strains. The statistic indexes associated with these two candidates show that they can noticeably affect the retaining wall deflections and are not strongly correlated with each other. The stiffness parameters $G_{0}, E_{\text {oed }}$, and $E_{\text {ur }}$ are indirectly optimized as we assume that they keep constant proportions to $E_{50}$, while other parameters are not changed as they have relatively insignificant influences on the deformations of excavation support structures. The field measurement entering into the inverse modelling is the retaining wall deflections corresponding to the excavation depth of $13 \mathrm{~m}$ (i.e., observation 


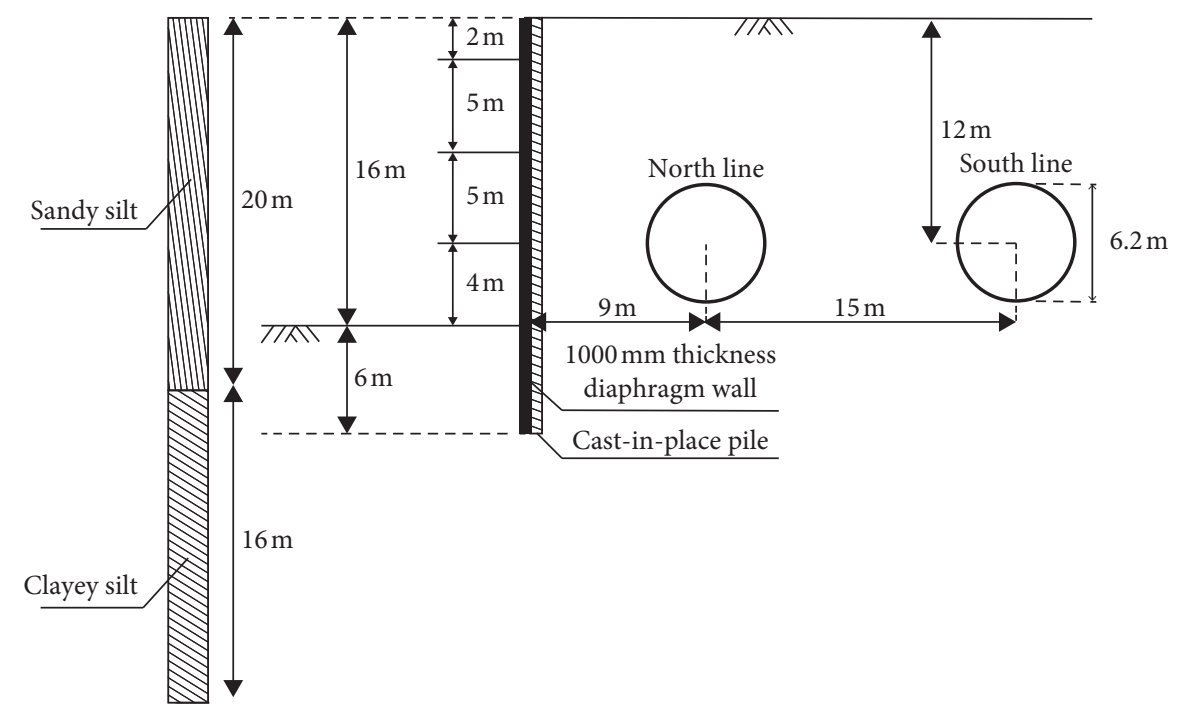

Figure 5: Schematics showing the excavation and tunnel geometries in the case study of Hangzhou, China.

TABLE 4: Mechanical properties of excavation support and tunnel structures in the case study of Hangzhou, China.

\begin{tabular}{|c|c|c|c|c|c|c|c|}
\hline \multicolumn{3}{|c|}{ Retaining wall } & \multirow{2}{*}{$\begin{array}{c}\text { Strut } \\
E A\left(10^{6} \mathrm{kN}\right)\end{array}$} & \multicolumn{4}{|c|}{ Tunnel } \\
\hline $\begin{array}{l}E I \\
\left(10^{6} \mathrm{kN} \times \mathrm{m}^{2} / \mathrm{m}\right)\end{array}$ & $\begin{array}{c}E A \\
\left(10^{6} \mathrm{kN} / \mathrm{m}\right)\end{array}$ & Poisson's ratio & & Diameter $(\mathrm{m})$ & Thickness (m) & Young's modulus (GPa) & Poisson's ratio \\
\hline 8.44 & 45 & 0.2 & 21.6 & 6.2 & 0.35 & 28 & 0.2 \\
\hline
\end{tabular}

TABLE 5: Soil parameters before and after inverse analysis.

\begin{tabular}{|c|c|c|c|c|}
\hline \multirow{2}{*}{ Stratum } & \multicolumn{2}{|c|}{ Original } & \multicolumn{2}{|c|}{ Optimized } \\
\hline & Sandy silt & Clayey silt & Sandy silt & Clayey silt \\
\hline Thickness (m) & 20 & 16 & 20 & 16 \\
\hline Unit weight $\left(\mathrm{kN} / \mathrm{m}^{3}\right)$ & 19.3 & 18.9 & 19.3 & 18.9 \\
\hline$E_{50}^{\mathrm{ref}}(\mathrm{MPa})$ & 15.00 & 9.00 & 3.09 & 1.85 \\
\hline$E_{\text {oed }}^{\text {rof }}(\mathrm{MPa})$ & 15.00 & 9.00 & 3.09 & 1.85 \\
\hline$E_{\text {ur }}^{\text {ref }}(\mathrm{MPa})$ & 60.00 & 50.00 & 12.36 & 10.3 \\
\hline$G_{0}^{\mathrm{rr} f}(\mathrm{MPa})$ & 200.00 & 184.00 & 41.19 & 37.9 \\
\hline$\gamma_{0.7}$ & $2.00 \times 10^{-4}$ & $4.00 \times 10^{-4}$ & $4.12 \times 10^{-5}$ & $8.23 \times 10^{-5}$ \\
\hline$c(\mathrm{kPa})$ & 5 & 6 & 5 & 6 \\
\hline$\varphi\left({ }^{\circ}\right)$ & 34 & 18.5 & 34 & 18.5 \\
\hline$M$ & 0.8 & 0.8 & 0.8 & 0.8 \\
\hline
\end{tabular}

from the earlier stage of construction). The optimized soil parameters are listed in Table 3.

The ANN predictions based on those updated soil parameters are included in Figure 6. The results clearly show that the soil parameters optimized by solely using the observations from early construction stages also lead to a good prediction of the response at later and more critical stages of construction. Moreover, Figure 6 emphasizes that, with a reasonable set of soil parameters, the trained ANN model can reasonably represent the deformations of the earth retaining wall.

By inputting the maximum deflections of earth retaining wall into the mechanistic model, the response of the two tunnels next to the excavation can be computed. The measured vertical and horizontal displacements of the tunnels that correspond to the excavation depth of $16 \mathrm{~m}$ are shown in Figure 7 (only the maximum displacements are reported), together with results obtained by using the proposed analytical model. The mechanical and geometrical properties of the tunnel structure used in the computation are listed in Table 3. Moreover, the length of the tunnels is set to $1000 \mathrm{~m}$ so that the shear forces and moments acting on both ends of the tunnels can be neglected. Young's modulus and Poisson's ratio for the soils surrounding the tunnels (i.e., see equation (3)) are converted from the optimized $G_{0}$ (considering the rather small soil movements around the tunnel) and $v_{\text {ur }}$ of the top sandy silty layer, i.e., where the tunnel is embedded.

It is seen from Figure 7 that three types of maximum retaining wall deflections are used to compute tunnel 


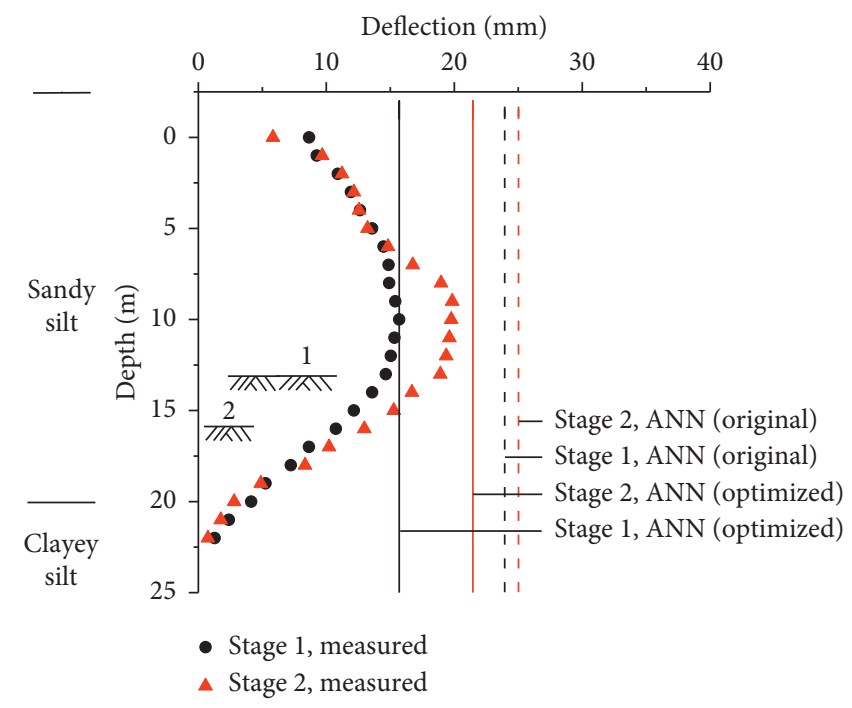

Figure 6: Comparison between measured retaining wall deflections in the case study of Hangzhou, China, and those predicted by the ANN model. Ground surface symbol shows the excavation depths corresponding to the two stages (i.e., stage 1: excavate to $13 \mathrm{~m}$; stage 2 : excavate to $16 \mathrm{~m}$ ).

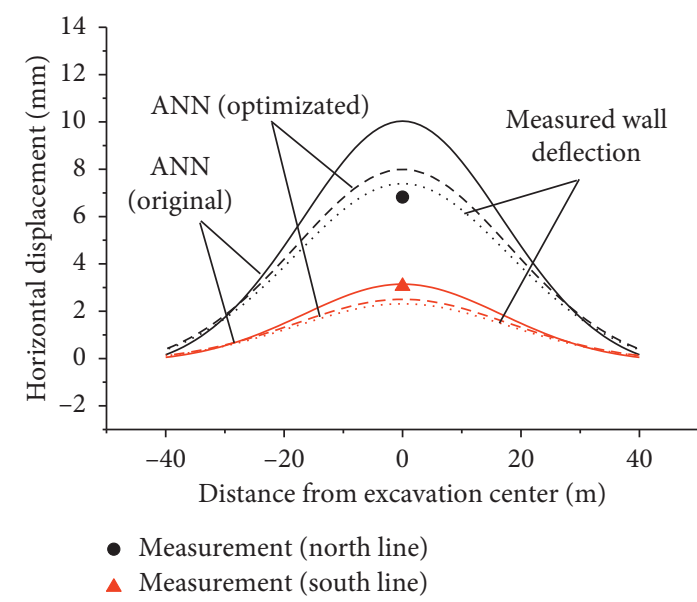

(a)

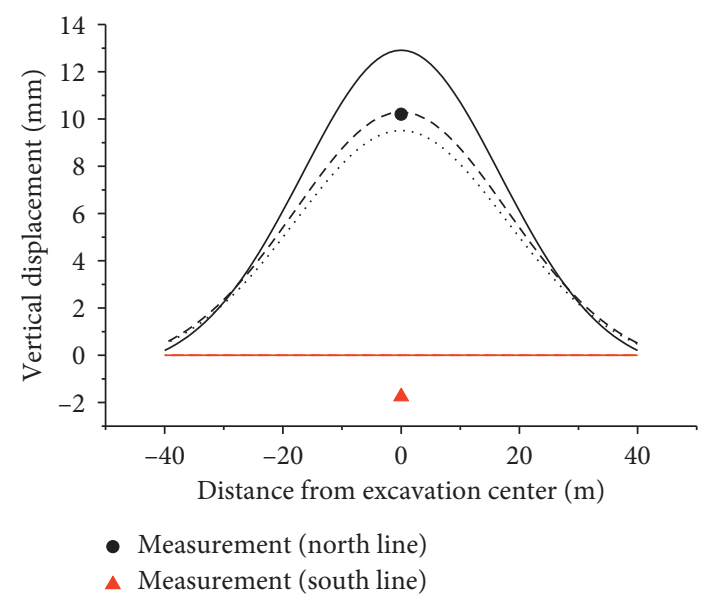

(b)

FIGURE 7: Comparison between measured and predicted tunnel displacements caused by adjacent excavation.

displacements, including the actual field measurements and ANN predictions based on the original and optimized soil parameters. The first observation from Figure 7 is that the computed results based on the measured wall deflection agree well with the measured tunnel response, thus suggesting that the mechanistic model can reasonably predict the tunnel displacements provided that the retaining wall deflection is accurately determined. By comparing the tunnel response computed by using the ANN predictions with the original and optimized soil parameters, the adaptivity and learning ability achieved by collaborating two machine learning techniques is emphasized in that by collecting observations at early stages of the excavation project, and the soil characterization updated by inverse modelling can form the basis of a new ANN prediction of the retaining wall deformations and consequently gradually improve the prediction regarding the response of adjacent tunnels.

\section{Conclusion}

An accurate prediction of the response of adjacent tunnels is critical for the plan, design, and construction of deep excavations in congested urban areas. The uncertainty and variability inherent to the subsurface compositions, extent, and behaviour and the nonlinear interactions between multiple agents (e.g., excavation support structures, soil strata, and tunnel structures) are the two primary resources leading to the complexity and difficulty of this task. This paper proposes an initiative to tackle this problem using process-based modelling, where information extracted from the construction processes of deep excavation is used to recognize the interaction patterns between multiple agents and to reduce the uncertainty associated with subsurface conditions, such that the response of tunnels induced by adjacent deep excavations can be accurately predicted. To 
proof the concept, we propose a simple process-based model composed of mechanistic module, artificial neural network (ANN) module, and inverse modelling module. These components are specialized in addressing particular challenging aspects of the targeted engineering problem but are interrelated forming a collaborative group. Specifically, the mechanistic component is responsible for computing the response of tunnel in accordance with the deflections of earth retaining wall. To predict the latter information, the ANN model is built aimed at learning and recognizing the patterns involved in the interactions between excavation geometries, excavation support structures, and soil properties. The inverse modelling module is included to reduce the uncertainty associated with soil parameters entering into the ANN model, which enables automatic learning and update of soil properties with the accumulation of information generated during construction processes. The main conclusions that can be drawn from this work include the following:

(1) While being trained by synthetic samples, the ANN model can reasonably predict the maximum deflections of retaining wall measured in actual engineering projects.

(2) The mechanistic model can reasonably predict the displacements of tunnels caused by adjacent excavations provided that the deflections of earth retaining wall can be accurately estimated.

(3) The quality of the ANN prediction highly depends on the characterization of soil parameters. Inverse modelling based on the ANN model can reduce the uncertainty associated with soil parameters and consequently improves the prediction of retaining wall deformations and tunnel displacements.

Lastly, it should be noted that the work presented here merely represents the first step towards accurately predicting the influences of deep excavations on adjacent tunnels by harnessing the power of process-based modelling. Despite the promising features exhibited by the ANN model trained by synthetic cases, the information obtained from real-world engineering projects is indispensable and the best dataset for training the model. For this purpose, next step that may be undertaken is to integrate the proposed model into BIM platforms such that (1) the ANN model can be trained by big data collected from actual deep excavation projects, (2) the uncertainty associated with site-specific soil properties can be reduced by inverse modelling with the accumulation of field observations, and (3) deep excavations can be managed dynamically and adaptively to protect nearby tunnels.

\section{Data Availability}

The data of field measurements, results generated by finite element simulations, and the proposed analytical model, which are used to support the findings of this study, are included within the article. The entire dataset of samples used for training the ANN model is available from the corresponding author upon request.

\section{Conflicts of Interest}

The authors declare that there are no conflicts of interest regarding the publication of this paper.

\section{Acknowledgments}

This study was supported by the Shanghai Science and Technology Committee Rising-Star Program (19QC1400500) and Fundamental Research Funds for the Central Universities.

\section{References}

[1] M. Doležalová, "Tunnel complex unloaded by a deep excavation," Computers and Geotechnics, vol. 28, no. 6-7, pp. 469-493, 2001.

[2] Z. Hu, Z. Yue, J. Zhou, and L. G. Tham, "Design and construction of a deep excavation in soft soils adjacent to the Shanghai metro tunnels," Canadian Geotechnical Journal, vol. 40, no. 5, pp. 933-948, 2003.

[3] S. Shen, H. Wu, Y. Cui, and Z. Yin, "Long-term settlement behaviour of metro tunnels in the soft deposits of Shanghai," Tunnelling and Underground Space Technology, vol. 40, pp. 309-323, 2014.

[4] J. Zhang, J. Chen, J. Wang, and Y. Zhu, "Prediction of tunnel displacement induced by adjacent excavation in soft soil," Tunnelling and Underground Space Technology, vol. 36, pp. 24-33, 2013.

[5] C. Chang, C. Sun, S. Duann, and R. Hwang, "Response of a taipei rapid transit system (TRTS) tunnel to adjacent excavation," Tunnelling and Underground Space Technology, vol. 16, no. 3, pp. 151-158, 2001.

[6] X. Huang, H. F. Schweiger, and H. Huang, "Influence of deep excavations on nearby existing tunnels," International Journal of Geomechanics, vol. 13, no. 2, pp. 170-180, 2001.

[7] M. Li, J. Chen, J. Wang, and Y. Zhu, "Comparative study of construction methods for deep excavations above shield tunnels," Tunnelling and Underground Space Technology, vol. 71, pp. 329-339, 2018.

[8] S. Azhar, "Building information modeling (BIM): trends, benefits, risks, and challenges for the AEC industry," Leadership and Management in Engineering, vol. 11, no. 3, pp. 241-252, 2011.

[9] R. Volk, J. Stengel, and F. Schultmann, "Building information modeling (BIM) for existing buildings-literature review and future needs," Automation in Construction, vol. 38, pp. 109127, 2014.

[10] I. A. Basheer and M. Hajmeer, "Artificial neural networks: fundamentals, computing, design, and application," Journal of Microbiological Methods, vol. 43, no. 1, pp. 3-31, 2000.

[11] A. K. Jain, J. Jianchang Mao, and K. M. Mohiuddin, "Artificial neural networks: a tutorial," Computer, vol. 29, no. 3, pp. 31-44, 1996.

[12] E. P. Poeter and M. C. Hill, "Inverse models: a necessary next step in ground-water modeling," Ground Water, vol. 35, no. 2, pp. 250-260, 1997.

[13] R. J. Finno and M. Calvello, "Supported excavations: observational method and inverse modeling," Journal of Geotechnical and Geoenvironmental Engineering, vol. 131, no. 7, pp. 826-836, 2005.

[14] L. Mu, R. J. Finno, M. Huang, T. Kim, and K. Kern, "Defining the soil parameters for computing deformations caused by 
braced excavation," Maejo International Journal of Science and Technology, vol. 9, no. 2, pp. 165-180, 2015.

[15] K. J. Xu and H. G. Poulos, "3-D elastic analysis of vertical piles subjected to "passive" loadings," Computers and Geotechnics, vol. 28 , no. 5, pp. 349-375, 2001.

[16] N. Loganathan, H. G. Poulos, and K. J. Xu, "Ground and pilegroup responses due to tunnelling," Soils and Foundations, vol. 41, no. 1, pp. 57-67, 2001.

[17] L. Mu, M. Huang, and R. J. Finno, "Tunnelling effects on lateral behavior of pile rafts in layered soil," Tunnelling and Underground Space Technology, vol. 28, no. 1, pp. 192-201, 2012.

[18] A. Klar, T. E. B. Vorster, K. Soga, and R. J. Mair, "Soil-pipe interaction due to tunnelling: comparison between Winkler and elastic continuum solutions," Géotechnique, vol. 55, no. 6, pp. 461-466, 2005.

[19] R. J. Jardin, D. M. Potts, A. B. Fourie, and J. B. Burland, "Studies of the influence of non-linear stress-strain characteristics in soil-structure interaction," Geotechnique, vol. 36, no. 3, pp. 377-396, 1986.

[20] J. H. Atkinson and G. Sallfors, "Experimental determination of stress-strain-time characteristics in laboratory and in situ tests," in Proceedings of the 10th European Conference on Soil Mechanics and Foundation Engineering, A. A. Balkema, Rotterdam, Netherlands, pp. 915-956, May 1991.

[21] J. H. Atkinson, "Non-linear soil stiffness in routine design," Géotechnique, vol. 50, no. 5, pp. 487-508, 2000.

[22] L. Mu and M. Huang, "Small strain based method for predicting three-dimensional soil displacements induced by braced excavation," Tunnelling and Underground Space Technology, vol. 52, pp. 12-22, 2016.

[23] J. Yu, C. Zhang, and M. Huang, "Soil-pipe interaction due to tunnelling: assessment of Winkler modulus for underground pipelines," Computers and Geotechnics, vol. 50, pp. 17-28, 2013.

[24] T. Benz, Small-strain stiffness and its numerical consequences, Ph.D. thesis, University of Stuttgart, Stuttgart, Germany, 2007.

[25] S. Likitlersuang, C. Surarak, D. Wanatowski, E. Oh, and A. Balasubramaniam, "Finite element analysis of a deep excavation: a case study from the Bangkok MRT," Soils and Foundations, vol. 53, no. 5, pp. 756-773, 2013.

[26] S. Liao, S. Wei, and S. Shen, "Structural responses of existing metro stations to adjacent deep excavations in Suzhou, China," Journal of Performance of Constructed Facilities, vol. 30, no. 4, Article ID 04015089, 2015.

[27] J. Yin, "Application of hardening soil model with small strain stiffness in deep foundation pits in Shanghai," Chinese Journal of Geotechnical Engineering, vol. 32, pp. 166-172, 2010, in Chinese.

[28] Y. Shi, J. Ruan, and C. Wu, "Xiamen area typical stratum of HS-small model for small strain parameters sensitivity analysis," Science Technology and Engineering, vol. 17, no. 2, pp. 105-110, 2017, in Chinese.

[29] F. Liu, "Influence on adjacent tunnel in process of deep foundation," Master's thesis, Zhejiang University, Zhejiang, China, 2015, in Chinese.

[30] K. Levenberg, "A method for the solution of certain nonlinear problems in least squares," Quarterly of Applied Mathematics, vol. 2, no. 2, pp. 164-168, 1944.

[31] D. W. Marquardt, "An algorithm for least-squares estimation of nonlinear parameters," Journal of the Society for Industrial and Applied Mathematics, vol. 11, no. 2, pp. 431-441, 1963.
[32] H. Zhou, "Study on the influence of excavation to adjacent existing tunnels in sandy silt area," Master's thesis, Zhejiang University of Technology, Zhejiang, China, 2015, in Chinese. 